\title{
Sequence based analysis of U-2973, a cell line established from a double-hit B-cell lymphoma with concurrent MYC and BCL2 rearrangements
}

\author{
Sean D Hooper ${ }^{1,2}$, Xiang Jiao ${ }^{1}$, Elisabeth Sundström¹, Farah L Rehman², Christian Tellgren-Roth', \\ Tobias Sjöblom ${ }^{1}$ and Lucia Cavelier ${ }^{1 *}$
}

\begin{abstract}
Background: Double-hit lymphoma is a complex and highly aggressive sub-type of B-cell lymphoma, which has recently been classified and is an area of active research interest due to the poor prognosis for patients with this disease. It is characterized by the presence of both an activating MYC chromosomal translocation and a simultaneous additional oncogenic translocation, often of the BCL2 gene. Recently, a cell line was established from a patient with this complex lymphoma and analyzed using conventional tools revealing it contains both MYC and $B C L 2$ translocation events.

Findings: In this work, we reanalyzed the genome of the cell line using next generation whole genome sequencing technology in order to catalogue translocations, insertions and deletions which may contribute to the pathology of this lymphoma type.

Conclusions: We describe the cell line in much greater detail, and pinpoint the exact locations of the chromosomal breakpoints. We also find several rearrangements within cancer-associated genes, which were not found using conventional tools, suggesting that high throughput sequencing may reveal novel targets for therapy, which could be used concurrently with existing treatments.
\end{abstract}

Keywords: Double hit lymphoma, Sequencing, Chromosomal rearrangements

\section{Findings}

Rearrangements in the human genome are a common feature of B-cell lymphomas with recurrent reciprocal chromosomal translocations present in approximately $40 \%$ of cases [1]. Certain translocations are considered specific for certain types of lymphoma. For example, $\mathrm{t}(8 ; 14)(\mathrm{q} 24 ; \mathrm{q} 32)$ or variant translocations leading to constitutive activation of the $M Y C$ transcription factor are a hallmark of Burkitt's lymphoma and are present in 8095\% of cases [2]. Furthermore, in follicular lymphomas, a characteristic translocation occurs between chromosomes 14 and $18(t(14 ; 18))$ which deregulates the apoptosis-inhibiting gene $B C L 2$ [3], resulting in a failure to respond to cell death signals. In rare cases, these

\footnotetext{
* Correspondence: lucia.cavelier@igp.uu.se

'Department of Immunology, Genetics and Pathology, Rudbeck Laboratory, Uppsala University, Uppsala 751 85, Sweden

Full list of author information is available at the end of the article
}

rearrangements are concurrent or sequential, and these cases are therefore sometimes referred to as Double Hit (DH) lymphoma. According to the most recent WHO guidelines these tumors are classified as "B cell lymphoma unclassifiable with features intermediate between DLBCL and BL [1]. Several cases of DH lymphoma have been described "(e.g. [4-6]) and have attracted research interest due to their unique cytogenetic features and relatively poor prognosis. Recently a stable B-cell line (U-2973) was established [7] from a DH lymphoma patient. This derived cell line does not necessarily represent the full clonality or tumorigenesis of the patient, but retains the main translocations initially identified in the patient. U-2973 was analyzed using conventional cytogenetic tools such as fluorescent in situ hybridization (FISH), G-band staining and multicolor FISH. These approaches showed concurrent translocations at $\mathrm{t}(8 ; 12)$ (q24;p12) and $\mathrm{t}(14 ; 18)(\mathrm{q} 32 ; \mathrm{q} 21)$. However, the primary

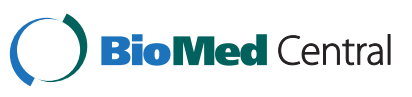


drawback of these methods is the low level of detail and the breakpoints of translocations can rarely be determined within tens or hundreds of kilobases. To address this issue, we have repeated the analysis of U-2973 with massive parallel sequencing on the SOLiD (ABI) platform. In short, DNA from U-2973 was sheared and fragments of on average $1 \mathrm{kbp}$ and $2.5 \mathrm{kbp}$ were selected for sequencing. By sequencing both ends of DNA fragments, we can capture not only translocations but also deletions and insertions with a high level of resolution. As a result we report a detailed map of chromosomal regions with extensive rearrangements not previously observed using conventional methods. We also pinpoint the DNA sequence at the breakpoint of $t(8 ; 12)$ and describe a transposition of DNA from chromosome 20 to chromosome 8 which could not be detected using conventional tools.

\section{Conventional analysis}

The M-FISH and karyotyping were performed as the DNA was extracted for the sequence analysis to verify the karyotype reported earlier (Additional file 1: Figure S1A and Additional file 2: Figure S2A) [7]. FISH hybridizations using gene specific probes ( $M Y C$ and $I G H-B C L 2)$ and whole chromosome paint hybridizations (WCP8 and WCP12; WCP 17 and WCP20) were performed to confirm the findings of the M-FISH, on cells from an independent culture. (Additional file 1: Figure S1B-D and Additional file 2: Figure S2B). The analysis showed basically the same karyotype as reported earlier with some discrepancies, probably due to changes during cell culture namely the reported trisomy 13, which we did not detect and a rearranged chromosome 20, that contained extra material from chromosome 17, which was earlier reported as containing material from chromosome 19. All cells analyzed showed the same karyotype in two independent cultures indicating the presence of a single clone in the sample used for DNA extraction and relative stability of the cell line. However we cannot confirm that the cell line faithfully represents all the lymphoma cells in the patient at diagnosis, but it rather constitutes a single clone selected when establishing the cell line in culture. The karyotype was further verified by SNP6 microarray analysis, which did not detect any trisomy 13 and showed additional 17-q material probably corresponding to the fragment incorporated into chromosome 20.

\section{Detailed rearrangements}

Deletions are characterized by a loss of DNA on one or both alleles. Through sequencing, we discovered deletions in U-2973 not previously described. We find for instance exonic deletions in the potential oncogene PIGU [8,9], and intronic deletions in PRKCA, which is overexpressed in some Burkitt's lymphomas [10]. Regional intergenic deletions include $6.8 \mathrm{kbp}$ on chromosome 5 , $4.5 \mathrm{kbp}$ on chromosome 1 , and $7.2 \mathrm{kbp}$ on chromosome 8. For DNA gains, the major insertions are roughly $800 \mathrm{bp}$ into ANO3 on chromosome 11 and the protocadherin $P C D H 9$ on chromosome 13 . We also find two cases of inversions involving exonic material in NKAIN3 and TSPAN8, a cell surface antigen, which were found to be overexpressed in some human tumor cell lines [11].

The characteristic rearrangements of a DH lymphoma are the two chromosomal translocations involving $M Y C$, $B C L 2$ och IGH. The translocation breakpoints (Figure 1) on the derivative chromosome $8 \operatorname{der}(8) \mathrm{t}(8 ; 12)$ were sequenced using Sanger sequencing and located to nucleotide coordinate 128835920 on chr8; 13kbp downstream of $M Y C$ and $45 \mathrm{kbp}$ upstream of PVT1, and at position 25082917 on chr12; roughly $12 \mathrm{kbp}$ upstream of LRMP, which appears to be deleted as a consequence of the translocation. The reciprocal translocation $\operatorname{der}(12)$ was not sequenced, but is positioned at roughly $129189 \mathrm{kbp}$ on chr8 and at approximately $25189 \mathrm{kbp}$ on chr12. Thus, it appears that most of the CASC1 gene has been lost, although one copy is likely to remain on the third copy of chromosome 12 as is also the case for LRMP. Most of the sequence of PVT1 has been lost from the genome, since there are no remaining copies of the original chromosome 8 .

The $t(14 ; 18)$ translocation is also validated by both libraries and PCR, and subsequently Sanger sequenced. One breakpoint lies within the IGH gene on chr14 and the other roughly $20 \mathrm{kbp}$ upstream of $B C L 2$ on chr18. This is consistent with previous observations of $I G H-B C L 2$ fusions in $96 \%$ of the blood sample [7] and in the FISH-analysis on the cell line (Additional file 1: Figure S1C). Furthermore, the breakpoint between chr14 and chr18 is interspersed with a 31 bp sequence which has no homology to the reference (hg18) genome. This sequence may have been extant in the genome before the translocation, but is more likely to have been inserted as a consequence of a non-homologous end joining of a double stranded chromosomal break.

In the previous analysis [7], it was established that the biallelic $\mathrm{t}(8 ; 12)$ translocation involved $M Y C$ on chr8 and potentially the $L R M P$ gene on chr12, but more detailed positions could not be determined. In this study, the biallelic translocation could be more accurately determined and the positions of the breakpoints suggest that LRMP may have been lost on the two derivative chromosomes 12. However, potential regulatory elements of LRMP may remain after the loss of sequence, and may deregulate other genes. There are no previously identified promoter regions or regulatory elements in this region, but it is interesting to note that the mouse ortholog to LRMP extends upstream to this 


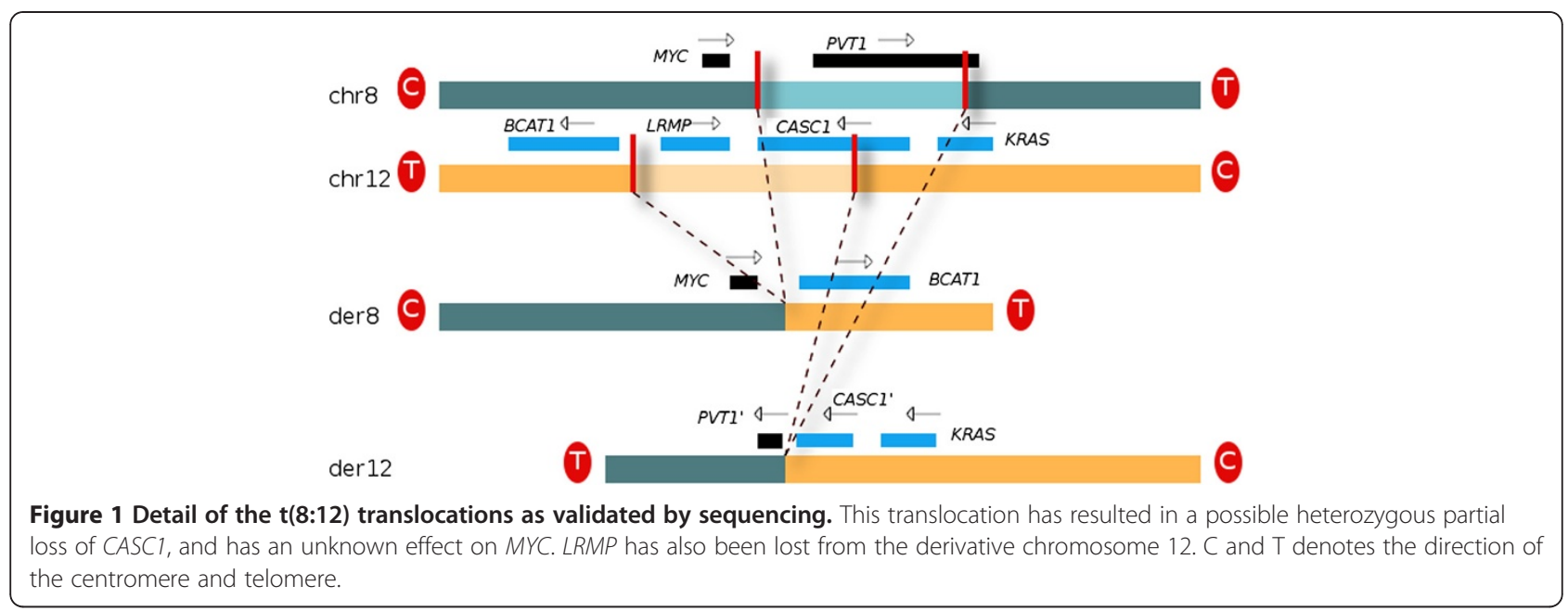

chromosomal location. Considering that LRMP is known to be expressed in germinal centre B-cells and in diffuse large B-cell Lymphomas of the "germinal" center subtype [12], we hypothesize that regulatory elements originally located upstream of the LRMP gene and juxtaposed to the $M Y C$-locus, might be involved in the de-regulation of $M Y C$. Interestingly, a recent report shows a case of a primary B-cell lymphoma with a $\mathrm{t}(8 ; 12)$ translocation also involving $M Y C$ and $L R M P$ [13]. Although the exact position of the breakpoint at $L R M P$ was not determined, FISH analysis showed that the 5' region of $L R M P$ was translocated to the $M Y C$ locus also in this case. Thus it might be of interest to determine the recurrence of $M Y C / L R M P$ rearrangements in a larger number of B-cell lymphomas.

Furthermore, the sequence analysis strongly suggests a translocation between chromosome 8 and 20. This translocation is supported by a large number of reads such as the previous translocations. However no $\mathrm{t}(8: 20)$ translocation could be observed by either FISH or karyotyping (see Additional file 2: Figure S2B). Since sequence reads could rather represent an insertion of DNA from one chromosome into the other, without otherwise disrupting chromosome 8 or 20, we designed primers considering the two possible insertion structures with a segment of 8 on 20 and vice versa. The PCR reactions thereafter confirmed that roughly $5 \mathrm{kbp}$ of intronic DNA within the VPS13B gene on chromosome 8 was inserted into chromosome 20 at position 58001229 and replacing some extant DNA including intronic sequences and exon 10 of the CDH6gene (Additional file 3: Figure S3). Whether or not this insertion has a driving role is unclear, but this case illustrates the sensitivity of matepair sequencing and also the need for a rigorous interpretation of the results.

Besides the three sequence-verified major translocation events, we also find other events with lower sequence read support and therefore rather represent transpositions of small regions of DNA, repetitive sequences or subclonal populations. The full list of translocations and affected genes is provided as Supplementary Material. This may be useful in identifying possible recurrent aberrations when compared to other lymphomas, and could also be informative when working with the U-2973 cell line. Thus comparisons to sequences from other lymphomas might clarify which of these events are recurrent and of pathogenic importance.

\section{Methods}

The U-2973 cell line was established from a biopsy with the informed consent of the patient and approved by the local ethical review board (Uppsala 00-275) as described in the original study [7]. DNA was prepared and sequenced on the ABI SOLiD parallel sequencing system according to the manufacturer's instructions. We prepared and sequenced two mate-pair libraries; one with an insert size of $2.5 \mathrm{kbps}$, and one with an insert size of $1.5 \mathrm{kbps}$. Thereafter, both ends of the fragment were ligated to adaptors. Using the Corona Lite software package (ABI), the 25bp reads were aligned to the human reference genome (NCBI build 36) and reads with unique matches on both ends were retained, resulting in a total of $119 \mathrm{M}$ read-pairs.

\section{Additional files}

Additional file 1: Figure S1A. G-band karyotype from the cell line retrieved from the double-hit cell lymphoma. 47,XY,der(8)t(8;12)(q24.21; p12.1) $\times 2,+12, \operatorname{der}(12) t(8 ; 12)(q 24.21 ; p 12.1) \times 2, t(14 ; 18)(q 32 ; q 21), \operatorname{der}(20) t(17 ; 20)$ $(q 23 ; q 13)$ chromosomes were harvested using a routine bone marrow protocol with one hour colcemide treatment. The slides were subsequently stained using standard Trypsin-Giemsa and karyotyped using the Cytovision software. S1B-D, FISH hybridization of chromosomes retrieved from the double-hit cell lymfoma. Chromosomes were 
harvested as above and hybridized with the MYC BA probe, the IGH-BCL2 DF probe (Abbott) and the WCP17 and WCP20 (Metasystems), according to the manufacturer's instructions. The pictures were retrieved using a Zeiss microscope and analyzed using the ISIS software.

Additional file 2: Figure S2. Multicolor FISH and WCP hybridizations. S2A. M-FISH hybridization showing the $t(8 ; 12), t(14 ; 18)$ and the $t(17 ; 20)$ translocations. S2B. WCP of chromosome 8 and 12, showing the two derived chromosomes 8 , the two derived chromosomes 12 and a normal chromosome 12

Additional file 3: Figure S3. Detail of an insertion of extragenous DNA and deletion of material on chromosome 20. This rearrangement was predicted by sequencing and undetected by conventional methods, but yet confirmed by PCR.

\section{Competing interests}

The authors declare no competing interests.

\section{Author contributions}

LC and SH conceived and designed the study. LC coordinated experiments and analysis. SH and CTR performed the bioinformatics analysis. LC performed the M-FISH analysis. All authors participated in discussions of different parts of the study. SH and ES wrote the manuscript. All authors read and approved the manuscript.

\section{Acknowledgments}

We thank Hans Ehrencrona, Helene Hällbook and Ola Söderberg for providing us with the cell line. We also acknowledge the staff at Uppsala Genome Center who performed the SOLiD sequencing, the staff at Clinical Genetics for the DNA sample preparation and help with the FISH-analysis, and Hanna G Kultima and the Uppsala array platform for performing the array hybridisations and analysis. Financial support for this project has been obtained from the Swedish research council VR (LC) and Clinical Genetics Department at Rudbeck Laboratory in Uppsala (SH).

\section{Author details}

'Department of Immunology, Genetics and Pathology, Rudbeck Laboratory, Uppsala University, Uppsala 751 85, Sweden. ${ }^{2}$ The Breakthrough Breast Cancer Research Centre, Institute of Cancer Research, Mary-Jean Mitchell Green Building, Chester Beatty Laboratories, Fulham Road, London SW3 6JB, UK.

Received: 3 June 2012 Accepted: 9 November 2012

Published: 22 November 2012

\section{References}

1. Aukema SM, Siebert R, Schuuring E, van Imhoff GW, Kluin-Nelemans HC, Boerma EJ, Kluin PM: Double-hit B-cell lymphomas. Blood 2011, 117(8):2319-2331.

2. Molyneux EM, Rochford R, Griffin B, Newton R, Jackson G, Menon G, Harrison CJ, Israels T, Bailey S: Burkitt's lymphoma. Lancet 2012, 379(9822):1234-1244.

3. Tsujimoto Y, Cossman J, Jaffe E, Croce CM: Involvement of the bcl-2 gene in human follicular lymphoma. Science 1985, 228(4706):1440-1443.

4. Pegoraro L, Palumbo A, Erikson J, Falda M, Giovanazzo B, Emanuel BS, Rovera G, Nowell PC, Croce CM: A 14;18 and an 8;14 chromosome translocation in a cell line derived from an acute B-cell leukemia. Proc Natl Acad Sci USA 1984, 81(22):7166-7170.

5. Mufti GJ, Hamblin TJ, Oscier DG, Johnson S: Common ALL with pre-B-cell features showing $(8 ; 14)$ and $(14 ; 18)$ chromosome translocations. Blood 1983, 62(5):1142-1146.

6. Kanungo A, Medeiros $L$, Abruzzo LV, Lin P: Lymphoid neoplasms associated with concurrent $\mathrm{t}(14 ; 18)$ and $8 \mathrm{q} 24 / \mathrm{c}-\mathrm{MYC}$ translocation generally have a poor prognosis. Mod Pathol 2006, 19(1):25-33.

7. Bostrom H, Leuchowius K, Hallbook H, Nordgren A, Thorn I, Thorselius M, Rosenquist R, Soderberg O, Sundstrom C: U-2973, a novel B-cell line established from a patient with a mature B-cell leukemia displaying concurrent $\mathrm{t}(14 ; 18)$ and MYC translocation to a non-IG gene partner. Eur J Haematol 2008, 81(3):218-225.

8. Guo Z, Linn JF, Wu G, Anzick SL, Eisenberger CF, Halachmi S, Cohen Y, Fomenkov A, Hoque MO, Okami K, et al: CDC91L1 (PIG-U) is a newly discovered oncogene in human bladder cancer. Nat Med 2004, 10(4):374-381.

9. Hong Y, Ohishi K, Kang JY, Tanaka S, Inoue N, Nishimura J, Maeda Y, Kinoshita T: Human PIG-U and yeast Cdc91p are the fifth subunit of GPI transamidase that attaches GPI-anchors to proteins. Mol Biol Cell 2003, 14(5):1780-1789.

10. Kamimura K, Hojo H, Abe M: Characterization of expression of protein kinase $\mathrm{C}$ isozymes in human B-cell lymphoma: relationship between its expression and prognosis. Pathol lnt 2004, 54(4):224-230.

11. Szala S, Kasai Y, Steplewski Z, Rodeck U, Koprowski H, Linnenbach AJ: Molecular cloning of cDNA for the human tumor-associated antigen CO-029 and identification of related transmembrane antigens. Proc Nat Acad Sci USA 1990, 87(17):6833-6837.

12. Tedoldi S, Paterson JC, Cordell J, Tan SY, Jones M, Manek S, Dei Tos AP, Roberton H, Masir N, Natkunam Y, et al: Jaw1/LRMP, a germinal centre-associated marker for the immunohistological study of B-cell lymphomas. J Pathol 2006, 209(4):454-463.

13. Singh RR, Ben-Neriah S, Johnson NN, Connors JM, Gascoyne RD, Horsman DE: Identification of a novel non-immunoglobulin/MYC translocation $\mathrm{t}(8 ; 12)(q 24 ; p 12)$ involving the LRMP gene in a primary B-cell lymphoma. A case report. Leuk Res 2012, 36(1):e22-e24.

\section{doi:10.1186/1756-0500-5-648}

Cite this article as: Hooper et al:: Sequence based analysis of U-2973, a cell line established from a double-hit B-cell lymphoma with concurrent MYC and BCL2 rearrangements. BMC Research Notes 2012 5:648.

\section{Submit your next manuscript to BioMed Central and take full advantage of:}

- Convenient online submission

- Thorough peer review

- No space constraints or color figure charges

- Immediate publication on acceptance

- Inclusion in PubMed, CAS, Scopus and Google Scholar

- Research which is freely available for redistribution 